\title{
LA FRAGMENTACIÓN PARLAMENTARIA NACIONAL Y LA REFORMA DEL SISTEMA ELECTORAL DE LA REGIÓN DE MURCIA: ¿NUEVAS OPORTUNI- DADES PARA UN PARTIDO REGIONALISTA?
}

The national parliamentary fragmentation and the reform of the electoral system of the Region of Murcia: new opportunities for a regionalist party?

\author{
DAVID PARRA GÓMEZ \\ Universidad de Murcia \\ david.parra@um.es
}

Resumen: La fragmentación parlamentaria tras las elecciones generales de 2016, marcada por el descenso de los dos grandes partidos mayoritarios y la irrupción de nuevas formaciones políticas, ha hecho aún más patente la necesidad de contar con el apoyo de partidos políticos de ámbito autonómico a la hora de sacar adelante iniciativas políticas de carácter nacional, así como el enorme rédito que tal apoyo supone para los territorios donde de tales partidos cuentan con soporte electoral. Asimismo, la composición fragmentada de la Asamblea Regional de Murcia fruto de las elecciones autonómicas de 2015, en las que el PP perdió la mayoría absoluta que ostentaba, trajo consigo una trascendental reforma de la ley electoral murciana. Ambos factores han favorecido la creación y expectativas de éxito de nuevos partidos regionalistas en la Región de Murcia, cuyos intentos por tener un espacio en el sistema de partidos murciano, sin embargo, han resultado infructuosos por el momento.

\begin{abstract}
The parliamentary fragmentation after the general elections of 2016, marked by the decline of the two major major parties and the emergence of new political formations, has made even more evident the need to count on the support of regional political parties at the time of take forward political initiatives of a national nature, as well as the enormous revenue that such support supposes for the territories where these parties have electoral support. Also, the fragmented composition of the Regional Assembly of Murcia fruit of the regional elections of 2015, in which the PP lost the absolute majority that it held, brought with it a transcendental reform of the electoral law of Murcia. Both factors have favored the creation and expectations of success of new regionalist parties in the Region of Murcia, whose attempts to have a space in the regional party system, however, have been unsuccessful for the moment.
\end{abstract}

Palabras clave: Parlamento; Reforma electoral; Partidos politicos.

Keywords: Parliament; Electoral reform; Political parties. 


\section{INTRODUCCIÓN.}

A punto de celebrar el 40 aniversario de la entrada en vigor de la Constitución de 1978, puede afirmarse que el sistema electoral español ha dado sobrada respuesta a algunos de los objetivos que el constituyente se propuso: (i) limitar la presencia de un excesivo número de partidos y coaliciones en las Cortes Generales; (ii) favorecer una representación reforzada de las provincias más despobladas; (iii) primar a los partidos que reciben los mayores apoyos en las distintas circunscripciones electorales (las provincias); y (iv) penalizar a los partidos más pequeños de ámbito nacional (aquellos que reciben menores apoyos electorales y no obtienen un voto muy concentrado en las circunscripciones provinciales). Los efectos de aquel sistema son bien conocidos:

En primer lugar, la consolidación de dos grandes formaciones políticas con implantación electoral en toda España, PSOE y PP, que han recibido, entre ambos, unos apoyos electorales y un número de escaños en el Congreso de los Diputados que, en cómputo total, se sitúan por encima del $80 \%$ hasta las elecciones de 20 de diciembre de 2015, si bien a partir de ese momento bajan al $60 \%$. En el Senado, la cifra de escaños electivos que suman entre ambos partidos es aún mayor.

En segundo término, los partidos de ámbito nacional que no obtienen fuertes apoyos electorales (terceras y cuartas fuerzas políticas en términos electorales) se ven drásticamente penalizados. Éste fue el caso del PSP hasta su integración en el PSOE, del CDS hasta su desaparición y del PCE y sus posteriores fórmulas políticas IU y Unidad Popular-IU hasta 2015, así como de UPyD en las elecciones generales de 2011y de PACMA en las de 2015 y 2016. Y, en tercer lugar, los partidos políticos de ámbito autonómico (regionalistas y nacionalistas), con voto concentrado en sus circunscripciones electorales, también obtienen buenos resultados en términos de escaños ${ }^{1}$, aun no siendo relevantes en términos de votos a nivel nacional, lo que les permite una posición muy relevante en las Cortes desde el punto de vista de la negociación política.

La formación e institucionalización de las Comunidades Autónomas ha dado lugar a la aparición de nuevos incentivos para la formación de partidos de ámbito autonómico con programas regionalistas o nacionalistas. Y ello principalmente, como remarca Paniagua (2008), por los estímulos derivados de su posición en el Parlamento español, especialmente en la Cámara Baja, provenientes de su función de "bisagra" para formar mayorías de distinto signo cuando las urnas no dan una mayoría absoluta. Este mismo fenómeno -continúa diciendo dicho autor- ha impulsado también a algunos de los partidos de ámbito nacional a subrogarse en discursos y propuestas propias del mercado político de los particularismos e incluso la adopción de estructuras de poder territorial en las que lo local es componente de casi mayor relevancia programática y discursiva por lo general. Éste es el caso de IU, del PSOE y, hasta ahora con menor énfasis, del $\mathrm{PP}^{2}$.

\footnotetext{
I. Desde la Legislatura constituyente hasta la XII, la media de escaños obtenida por partidos y coaliciones de corte nacionalista y regionalista en la Cámara Baja es de 54,I5. Fuentes: elaboración propia a partir de la web del Congreso de los Diputados: http://www.congreso.es.

2. Paniagua, J. L. (2008): “Representación y peso político de los partidos nacionalistas en las Cortes Generales”, en Temas para el debate, núm. I62 (Ejemplar dedicado a: Democracia y legislación electoral), pp. 54-56.
} 
Con carácter general, los partidos de ámbito autonómico han ejercido de partidos necesarios para completar mayorías parlamentarias en momentos clave: votaciones de investidura, aprobación de los Presupuestos Generales del Estado y una larga lista de leyes y acciones del Gobierno -amén de la reciente aprobación de la primera moción de censura desde la restauración democrática en 1978-. La faceta positiva de dichos comportamientos es que esos partidos han incorporado sus demandas a la política nacional y han contribuido a la estabilidad de sucesivos Gobiernos. Por el contrario, esos mismos partidos (aunque no todos, ni siempre ni con la misma intensidad) han jugado a cobrar un precio político o económico.

Todos, sean o no partidos de Gobierno en sus Comunidades Autónomas y tengan o no grupo parlamentario propio, tienen un apoyo electoral reducido en comparación con los partidos pequeños de ámbito nacional. Y todos ellos operan en el espacio parlamentario nacional, cuando hay Gobiernos en minoría, como verdaderos actores con auténtica capacidad de veto. Estas circunstancias refuerzan la imagen de que ocupan un espacio desproporcionado y tienen un sobrepeso político que alarga sus dimensiones y sus capacidades de condicionar la política nacional muy por encima de lo que los votos les confieren en términos comparativos.La crítica, además, se completa con la constatación de que sus condiciones (el precio y el valor de sus apoyos) están, por lo general, orientadas por intereses particularistas $\mathrm{y}$, en algunos casos, por aspiraciones secesionistas.

Por otra parte, la devastadora crisis económica y los diversos escándalos de corrupción arrasaron la confianza de la ciudadanía en las instituciones representativas y de gobierno, desafección que se tradujo en una demanda de regeneración democrática a la vez que dio paso a la irrupción de nuevas fuerzas políticas en los ámbitos estatal y autonómicotras las elecciones de 2015. Por lo que se refiere al segundo de esos ámbitos, la fragmentación política en varias Asambleas autonómicas propició ab initio en el seno de las mismas un debate de calado sobre la necesidad de reformar aspectos esenciales del respectivo sistema electoral. Debate que respondía, en cierto modo, a la demanda ciudadana -de la que se habían hecho eco los nuevos partidos en sus respectivos programas electorales- sobre la necesidad de contarcon un sistema electoral más justo que diera igual valor al voto de los ciudadanos para elegir a sus representantes. No es de extrañar, pues, que nuevas fuerzas políticas o coaliciones de partidos que consiguieron acceder a los Parlamentos regionales en 2015 planteasen la reforma de algunos aspectos del sistema electoral que les pudiera excluir en el futuro de la representación parlamentaria, incluyendo tal demanda en los pactos llevados a cabo con las formaciones tradicionales para formar gobiernos autonómicos.

El paradigma de esta tendencia lo encontramos en la Región de Murcia, donde, tras las elecciones de mayo de 2015, el pacto entre C's y PP para investir a un presidente popular incluyó una reforma de la ley electoral en dirección opuesta a la que había defendido este último partido en la anterior legislatura autonómica. Dicha reforma, que se materializó en julio de 2015, ponía fin al carácter singular del sistema electoral murciano al rebajar la barrera electoral del 5\% al 3\% y reducir las cinco circunscripciones a una única circunscripción, estimulando, así, la aparición de nuevas fuerzas políticas de carácter regionalista en una comunidad donde los intentos del regionalismo por tener un espacio en el sistema de partidos han fracasado. 
Al análisis de la virtualidad de ambos factores (la ventajosa posición de los partidos de ámbito autonómico en un Congreso con Gobiernos en minoría y la reforma del sistema electoral murciano) como acicate de las expectativas electorales de formaciones regionalistas en Murcia dedicamos la presente investigación.

\section{EL PESO DECISIVO DE LOS PARTIDOS NACIONALISTAS Y REGIONALIS- TAS EN EL ESCENARIO POLÍTICO NACIONAL.}

\subsection{Desmontando el mito de su sobrerrepresentación.}

Suele apuntarse que uno de los principales inconvenientes del sistema electoral español ${ }^{3}$ es que los partidos nacionalistas y regionalistas están sobrerrepresentados en el Congreso de los Diputados. Sin embargo, lo cierto es que el tamaño de su representación es bastante proporcional con el número de votos obtenido. En efecto, si atendemos al cálculo de la diferencia, en puntos porcentuales, entre votos y escaños en las doce elecciones a la Cámara Baja celebradas desde 1977 a 2015, la sobrerrepresentación o infrarrepresentación de esta clase de partidos es pequeña, $\mathrm{y}$ depende principalmente de dos factores:

1. Del tamaño de las circunscripciones presentes en la concreta Comunidad Autónoma en la que concentran su voto, es decir, de si está formada fundamentalmente por circunscripciones pequeñas, o si en aquélla las circunscripciones medianas y grandes tienen una presencia importante.

2. Y de los efectos de lo que Montero y Riera (2009) han llamado el sesgo conservador de nuestro sistema electoral ${ }^{4}$, consecuencia de otorgar sobrerrepresentación a las provincias rurales -por la combinación entre el prorrateo desviado que introduce el mínimo de diputados asignado a cada circunscripción por la Constitución (dos escaños a cada provincia, excepto Ceuta y Melilla que reciben uno)- y el pequeño tamaño de la Cámara, y que viene a infrarrepresentar ligeramente a las circunscripciones medianas y grandes ${ }^{5}$.

En el primer caso, los efectos positivos del sesgo conservador provocarán que el partido de ámbito autonómico se vea ligeramente sobrerrepresentado. Así le sucede al PNV, pues al ser de magnitud media-baja las circunscripciones que forman el País Vasco (Álava elige 4 representantes, Vizcaya 8 y Guipúzcoa 6), su promedio de sobrerrepresentación hasta 2015 es de 0,35 \%

\footnotetext{
3. Un análisis detallado de algunos de los principales inconvenientes de nuestro sistema electoral se contiene en el Informe del Consejo de Estado sobre las propuestas de modificación del régimen electoral general de 24 de febrero de 2009 (vid., concretamente, las páginas 145 y ss.).

4. Montero, J.R. y Riera, P. (2009): "El sistema electoral español: cuestiones de desproporcionalidad y de reforma”, en Anuario de la Facultad de Derecho de la Universidad Autónoma de Madrid, núm. 13, p. 234.

5. El sesgo conservador puede ser incluido en lo que García Escudero (20I6) denomina los “efectos mecánicos" del sistema electoral. Este autor lleva a cabo una interesante distinción de los factores que actúan en lo que él llama "primer paso de las elecciones", es decir, cuando el ciudadano emite un voto a favor de una concreta lista en los términos en los que ha sido presentada: (i) el conjunto de clivajes existente (como el clivaje izquierda-derecha- y el clivaje centro-periferia); (ii) el devenir de la política y los factores externos que influyen en ella (como las encuestas preelectorales y el comportamiento de los diversos partidos); y (iii) el efecto psicológico del sistema electoral (el llamado voto estratégico, insincero o útil). Vid., García Escudero, J. 2016: "El sistema electoral español, su rendimiento y la actual conveniencia de su reforma", comunicación presentada al XII Congreso Español de Sociología “Grandes transformaciones sociales, nuevos desafíos para la Sociología", Gijón, 30 de junio, r y 2 de julio de 2016, pp. 25-29.
} 
(Gráfico 1). En el segundo, los efectos negativos del mencionado sesgo -que infrarrepresentan ligeramente a las circunscripciones medianas y grandes- provocarán que el partido se vea ligeramente infrarrepresentado. Es el caso de CIU/DiL (aunque tres de las circunscripciones que componen Cataluña, Gerona, Tarragona y Lérida, eligen 6, 6 y 4 representantes, respectivamente, Barcelona elige 31 escaños), cuya infrarrepresentación en el período analizado es de 0,1\% (Tabla 1).

Precisamente esa misma concentración de voto es la que perjudica a los partidos pequeños de ámbito nacional, que al repartir su voto de forma más o menos uniforme por todo el territorio, y a pesar de que llegan a reunir un porcentaje de voto significativo, se ven muy infrarrepresentados. Así ha pasado, por ejemplo, con PCE/IU/UP, que a pesar de que a lo largo de las doce elecciones analizadas llegan a reunir un porcentaje de voto significativo $(5,70 \%)$, han alcanzado un promedio de infrarrepresentación del 3,6\% (Tabla 1$)^{6}$.

Tabla 1. Diferencia entre votos y escaños en elecciones al Congreso 1977-2015

\begin{tabular}{|l|l|l|l|l|l|l|}
\hline Elecciones & UCD & AP/PP & PSOE & PCE/IU/UP & CiU/DiL & PNV \\
\hline 1977 & 12,7 & $-3,6$ & 4,4 & $-3,6$ & $-0,6$ & 0,7 \\
\hline 1979 & 13,2 & $-3,2$ & 4,2 & $-4,2$ & $-0,5$ & 0,4 \\
\hline 1982 & $-3,7$ & 4,2 & 9,6 & $-2,9$ & $-0,2$ & 0,4 \\
\hline 1986 & & 4,0 & 8,5 & $-2,6$ & 0,1 & 0,2 \\
\hline 1989 & & 4,8 & 10,4 & $-4,2$ & 0,1 & 0,2 \\
\hline 1993 & & 5,5 & 6,7 & $-4,5$ & 0,0 & 0,2 \\
\hline 1996 & & 5,8 & 2,7 & $-4,5$ & 0,0 & 0,1 \\
\hline 2000 & & 7,8 & 1,5 & $-3,2$ & 0,1 & 0,5 \\
\hline 2004 & & 4,6 & 4,3 & $-3,6$ & $-0,4$ & 0,4 \\
\hline 2008 & & 4,1 & 4,4 & $-3,2$ & $-0,1$ & 0,5 \\
\hline 2011 & & 8,5 & 2,6 & $-3,8$ & 0,3 & 0,1 \\
\hline 2015 & & 6,2 & 4,0 & $-3,1$ & 0,0 & 0,5 \\
\hline Promedio & 7,4 & 4,1 & 5,3 & $-3,6$ & $-0,1$ & 0,35 \\
\hline
\end{tabular}

A la misma conclusión llegamos si comparamos el reparto de escaños en el Congreso como resultado de las elecciones generales celebradas el 26 de junio de 2016 con el reparto de escaños que habría habido en dicha Cámarasi éste hubiera sido del todo proporcional (Tabla 2) o se hubiera calculado con la fórmula D’Hondt sobre una circunscripción única (Tabla 3) ${ }^{8}$.

6. Índice de desproporcionalidad utilizado: Lijphart. No obstante, sea cual sea el indicador que se utilice, la desproporcionalidad que arroja el sistema electoral que rige la elección del Congreso de los Diputados alcanza elevadas cuotas. En Ocaña /Oñate (1999): 233 se ofrece tabla con los valores resultantes de aplicar distintos índices de desproporcionalidad a los resultados de las 7 primeras elecciones generales.

7. La diferencia (en puntos porcentuales) se calcula, a partir de los resultados electorales proporcionados por el Ministerio del Interior, restando al porcentaje de escaños el porcentaje de voto. En verde se muestran los porcentajes de sobrerrepresentación, y en rojo los de infrarrepresentación. Fuente: Montero /Fernández /Rama (2016).

8. Abreviaturas de partidos/coaliciones mencionados en Introducción y en Gráficos I, 2 y 3. AP: Alianza Popular. BNGNÓS: Bloque Nacionalista Galego. CC: Coalición Canarias. CDC: Convergència Democràtica de Catalunya. CiU: 
En ambos casos, el número de escaños en exceso que han obtenido los partidos nacionalistas y regionalistas con el actual sistema electoral, en contra de lo que habitualmente se afirma, o simplemente no existe (casos de ERC-CATSÍ y CC-PNC), o no esmuy significativo (apenas un escaño en el resto de casos).

Tabla 2. Diferencia entre resultados 26J y resultados distribución proporcional

\begin{tabular}{|l|l|l|l|}
\hline Partido & Resultado 26 J & Proporcional & Diferencia \\
\hline PP & 137 & 117 & 20 \\
\hline PSOE & 85 & 80 & 5 \\
\hline UP & 71 & 75 & 4 \\
\hline C's & 32 & 46 & 14 \\
\hline ERC-CATSÍ & 9 & 9 & 0 \\
\hline CDC & 8 & 7 & 1 \\
\hline PNV & 5 & 4 & 1 \\
\hline EH Bildu & 2 & 3 & 1 \\
\hline CC-PNC & 1 & 1 & 0 \\
\hline PACMA & 0 & 4 & 4 \\
\hline REC 0 VERDES & 0 & 1 & 1 \\
\hline UPyD & 0 & 1 & 1 \\
\hline VOX & 0 & 1 & 1 \\
\hline BNG-NÓS & 0 & 1 & 1 \\
\hline
\end{tabular}

Tabla 3. Diferencia entre resultados 26J y resultados fórmulaD'Hondt /C. única9

\begin{tabular}{|l|l|l|l|}
\hline Partido & Resultado 26 J & D’Hondt / C. única & Diferencia \\
\hline PP & 137 & 119 & 18 \\
\hline PSOE & 85 & 81 & 4 \\
\hline UP & 71 & 76 & 5 \\
\hline C's & 32 & 47 & 15 \\
\hline ERC-CATSÍ & 9 & 9 & 0 \\
\hline CDC & 8 & 7 & 1 \\
\hline PNV & 5 & 4 & 1 \\
\hline EH Bildu & 2 & 2 & 0 \\
\hline
\end{tabular}

Convergència i Unió. C's: Ciudadanos Partido de la Ciudadanía. DiL: Democràcia i Llibertat. EH Bildu: Euskal Herria Bildu. ERC-CATSÍ: Esquerra Republicana de Catalunya-Catalunya Sí. IU: Izquierda Unida. PACMA: Partido Animalista. PCE: Partido Comunista de España. PNC: Partido Nacionalista Canario. PNV: Partido Nacionalista Vasco. PP: Partido Popular. PSOE: Partido Socialista Obrero Español. REC o VERDES: Recortes Cero-Grupo Verde. UP: Unidos Podemos. UPyD: Unión, Progreso y Democracia.

9. En verde se muestra la cantidad de escaños en exceso que ha obtenido el partido con respecto a los que le hubiera correspondido con un reparto que aplicara la fórmula $\mathrm{D}^{\prime} \mathrm{Hondt}$ en una circunscripción única. Y en rojo se indica el número de escaños que ha dejado de obtener. Fuente: Olazabal (2016). 


\begin{tabular}{|l|l|l|l|}
\hline CC-PNC & 1 & 1 & 0 \\
\hline PACMA & 0 & 4 & 4 \\
\hline REC 0 VERDES & 0 & 0 & 0 \\
\hline UPyD & 0 & 0 & 0 \\
\hline VOX & 0 & 0 & 0 \\
\hline BNG-NÓS & 0 & 0 & 0 \\
\hline
\end{tabular}

\subsection{El porqué de su influencia desproporcionada: crónica de un apoyo parlamentario muy rentable.}

Aunque el efecto de concentración del voto no influye de forma significativa en la representatividad/proporcionalidad de los partidos nacionalistas y regionalistas - pues, como acabamos de ver, sólo los sobrerrepresenta o infrarrepresentade forma muyligera-, la suma de aquel efecto y la inexistencia de terceras o cuartas fuerzas de ámbito nacional con un tamaño similar al de los partidos nacionalistas y regionalistas ha llevado a que éstos tengan una relevancia política desmesurada para su tamaño en escaños. El motivo lo explica con claridad Tena Arregui (2015): "Los partidos nacionalistas/regionalistas tiene una influencia desproporcionada en el Congreso. Ojo, no me refiero a que estén sobrerrepresentados desde un punto de vista de voto proporcional (porque en absoluto es así) sino que, como consecuencia de la sobrerrepresentación del PP y PSOE a costa de los partidos minoritarios de ámbito nacional, ellos son los que hacen de bisagra cuando uno de los grandes no obtiene mayoría absoluta"10.

Así pues, la influencia desproporcionada que los partidos políticos de ámbito autonómico - especialmente los nacionalistas- vienen teniendo en el Congreso de los Diputados no se debe a una prima electoral que no existe (su tamaño en escaños, insistimos de nuevo, es bastante proporcional con el número de votos obtenido), sino a su apoyo parlamentario a los dos grandes partidos nacionales -PSOE y PP-cuando éstos han necesitado alcanzar la mayoría en la CámaraBaja en algunos momentos políticos clave, de entre los que destacamos, tanto por su importancia política como por la indudable rentabilidad obtenida, los siguientes: a) La tercera investidura de Felipe González y quinta de la democracia se celebró los días 4 y 5 de diciembre de 1989 y estuvo precedida de dos acontecimientos importantes: la anulación de las elecciones en Pontevedra, Murcia y Melilla y la ausencia de los diputados de HerriBatasuna (HB). Los 166 votos socialistas no eran suficientes para que el Congreso de los Diputados, integrado el día 5 por 332 parlamentarios, eligiera en primera votación a Felipe González como presidente, una dificultad que soslayó gracias al respaldo del diputado tinerfeño Luis Mardones, por entonces en las filas de las Agrupaciones Independientes de Canarias (AIC $)^{11}$. Las condiciones negociadas por el PSOE para contar con el 'voto de Mardones' fueron terminar la red de carreteras de Canarias, incrementar la subvención al transporte de viajeros y mercancías, aumentar las partidas canarias en los Presupuestos Generales del Estado y la mejora del Régimen Económico y Fiscal (REF) isleño.

b) En 1993, aunque el PSOE volvió a ganar las elecciones por cuarta vez consecutiva, perdió la mayoría absoluta de la que disfrutaba hasta entonces, quedándose a 17 escaños de la Io. Tena Arregui, R. (20I5): "El sistema electoral". Serie sobre la reforma constitucional (IV). Recuperado de https:// hayderecho.com.

II. Uno de los partidos germen de Coalición Canaria. 
misma, con 159. Los socialistas negociaron el apoyo de los nacionalistas vascos y catalanes. CiU, con 17 escaños, votó "sí" a la investiduraa cambio arañar para Cataluña la llamada "corresponsabilidad fiscal" (la cesión del 15\% del IRPF) y el desarrollo pleno del Estatuto de Autonomía con las consiguientes transferencias. También el PNV, que había logrado 5 escaños y a quienes los socialistas llegaron a ofrecer incluso la cartera de Industria, apoyó la investidura de González, aunque rechazó entrar en su Ejecutivo.

c) El PP ganó por primera vez las elecciones el 3 de marzo de 1996, pero se quedó a 20 escaños de la mayoría absoluta. Se convertía en necesaria, de nuevo, la vía del diálogo y del pacto con nacionalistas y regionalistas para asegurar la gobernabilidad. Aznar logró el apoyo de CC, PNV y CiU, convirtiéndose en el cuarto jefe del Ejecutivo. Los compromisos acordados con CC fueron la reforma del Estatuto de Autonomía y la culminación de las trasferencias pendientes, tratar de conseguir un Estatuto Permanente para Canarias en la UE, la mejora del REF y la puesta en marcha de un Plan Integral de Empleo y de un Plan de Infraestructuras para Canarias. La lista de concesiones de Aznar a CiU fue larga ${ }^{12}$ : transferencias en diversas materias (competencias de tráfico a los Mossosd'Esquadra, justicia, educación, agricultura, cultura, farmacias, sanidad, empleo, puertos, medio ambiente, mediación de seguros, política lingüística y vivienda), eliminación de la figura del gobernador civil, que fue sustituida por el subdelegado del Gobierno, con menos competencias que el anterior, importantes inversiones en Cataluña(como las ampliaciones del puerto y del aeropuerto de Barcelona) y la cesión del $30 \%$ del IRPF ${ }^{13}$. En el caso del PNV, que tenía solo 5 diputados en 1996, el pacto incluyó un nuevo desarrollo del Concierto Económico (recaudación de impuestos de alcohol, tabaco y gasolinas), la transferencia de la formación continua, la devolución de patrimonio incautado en la Guerra Civil y el compromiso de negociar durante la legislatura el pleno desarrollo del Estatuto de Guernica.

d) En 2004, Zapatero llegó a La Moncloa gracias a los votos, entre otros, de ERC, $\mathrm{BNG}, \mathrm{CCy} \mathrm{CHA}^{14}$. Los canarios, gallegos y aragoneses exigieron básicamente por su apoyo nuevas transferencias y aumento de las inversiones estatales en sus respectivas Comunidades Autónomas. Pero fueron los nacionalistas catalanes quienes obtuvieron mayores ventajas de su apoyo al comprometerse el presidente socialista a aprobar un nuevo Estatuto y a respetar el texto salido del Parlamento catalán ${ }^{15}$. En la siguiente legislatura, el leonés se convirtió en el primer presidente de la democracia investido por mayoría simple, gracias a la abstención, entre otros, de PNV, CiU, ICV ${ }^{16}$, BNG y CC. Mediada la legislatura, PNV y CC se convirtieron en aliados preferentes al apoyar los presupuestos de 2010 y 2011. A cambio, los regionalistas canarios obtuvieron, entre otras contrapartidas, el reconocimiento de las aguas entre las islas del archipiélago como propias de la comunidad y no internacionales, la transferencia de competencias pendientes (como las políticas activas de empleo y la inspección

I2. Las negociaciones se concretaron en el pacto conocido como del Majestic por el hotel en el que se clausuraron. 13. Conviene apuntar que el PP también apartó a Aleix Vidal-Quadras de la presidencia del partido en Cataluña por expresa petición de Pujol.

14. Chunta Aragonesista.

15. El Estatuto catalán de 2006 fue declarado parcialmente inconstitucional por el Tribunal Constitucional (Sentencia $3 \mathrm{I} / 2010$, de 28 de junio de 2010). En concreto, el Alto Tribunal declaró inconstitucionales I4 artículos y sujetos a la interpretación del tribunal otros 27. Además, el TC estimó carentes de eficacia jurídica las referencias que se hacen en el preámbulo a Cataluña como nación.

I6. Iniciativa per Catalunya Verds. 
de trabajo) y el mantenimiento de la rebaja del $50 \%$ en los vuelos a los residentes canarios, además del compromiso de desbloquear la actualización del Estatuto de Autonomía canario. Los nacionalistas vascos, por su parte, lograron la transferencia de 20 de las 27 competencias que restaban para completar el Estatuto de Guernica ${ }^{17}$ y 112 millones de euros en inversiones adicionales para el País Vasco en el 2011. Y ese mismo año Zapatero autorizó a la Generalidad de Cataluña aumentar su nivel de endeudamiento para enjugar su déficit.

e) Aunque la radicalización del nacionalismo catalán iniciada en 2014 y, sobre todo, el cambio del formato del sistema de partidos que supuso la irrupción en 2015 de los llamados partidos de la "nueva política", Ciudadanos y Podemos ${ }^{18}$, presagiaban el fin del decisivo papel de los partidos autonómicos que venimos relatando, el bloqueo mutuo -a través de los respectivos vetos- entre los nuevos partidos es la causa de que la construcción de una mayoría parlamentaria haya seguido exigiendo el concurso nacionalista/regionalista. En efecto, Mariano Rajoy fue investido presidente en segunda votación ${ }^{19}$ gracias a los 170 votos a favor de PP, Cs, CC, FA ${ }^{20}$ y UPN ${ }^{21}$, y a las abstenciones de 68 diputados socialistas ${ }^{22}$. Y pronto se vio obligado a recurrir a los diputados nacionalistas y regionalistas para sacar adelante los Presupuestos Generales del Estado para 2017 y 2018, avalados en la Cámara Baja por PNV, FA, UPN, CC y NC ${ }^{23}$ a cambio, cómo no, de importantes beneficios a sus respectivas comunidades:

- CC negoció con el Gobierno una mejora en las inversiones estatales para Canarias y extraer el REF del sistema de financiación autonómica (para unirlo a los Presupuestos).

- NC, con un solo diputado ${ }^{24}$, logró bonificaciones del $75 \%$ para el transporte interinsular de pasajeros por mar y aire y del $100 \%$ para el de mercancías entre islas y de las Canarias a la península ${ }^{25}$, la eliminación del llamado "impuesto al Sol”26, la tramitación de

17. Además de las políticas activas de empleo, el Gobierno traspasó la Inspección de Trabajo, el tráfico marítimo en aguas vascas o las políticas de formación del Instituto Social de la Marina, además de permitir la presencia de técnicos de la hacienda foral vasca en las reuniones del Ecofin y cumplir con los plazos de la "Y" vasca en la parte que corresponde al Estado.

I8. Tras las elecciones generales de 20 de diciembre de 20I5, Podemos y Ciudadanos irrumpieron en el Congreso de los Diputados como tercera y cuarta fuerzas parlamentarias, poniendo fin al anterior bipartidismo imperfecto en el que PP y PSOE se repartían alrededor de 20 millones de votos. En el Senado la fragmentación fue menor, pues el PP logró mantener -sumando los senadores designados por las Asambleas autonómicas- la mayoría absoluta en esta Cámara.

19. La investidura se produjo transcurridos cuatro meses de las elecciones generales de junio de 2016, en las que el PP mejoró sus resultados en la Cámara Baja, aunque quedó lejos de la mayoría absoluta, mientras que Podemos y Ciudadanos sufrieron un leve descenso.

20. Foro Asturias.

2I. Unión del Pueblo Navarro.

22. Quince diputados socialistas, quebrantando la disciplina de voto, votaron en contra.

23. Nueva Canarias.

24. Pedro Quevedo, el veterano parlamentario de Nueva Canarias, se convirtió en el diputado i76 necesario para que el Gobierno de Mariano Rajoy solventara su primer examen serio de la legislatura: los Presupuestos Generales del Estado de 2017. Y ello a pesar de que concurrió a las elecciones en coalición con el PSOE y de votar no a la investidura de Rajoy. En las Islas Canarias algunos ya le ven como un "héroe" capaz de arañar importantes inversiones al Ejecutivo popular para su tierra. Vid., "Pedro Quevedo: botín y venganza del diputado canario" (29/05/20I7). Recuperado de: http://www.elmundo.es. 25. Que suponen una inyección de 65 millones. Bonificaciones que, además, se mantendrán en los próximos años, ya que fueron incorporadas como derechos adquiridos en la reforma de la Ley del REF que, a iniciativa del Ejecutivo, se aprobó en junio de 2017.

26. Que grava el autoconsumo de la energía que se produce desde los paneles fotovoltaicos y eólicos. 
una reforma del Estatuto de Autonomía canario y un acuerdo de financiación autonómica que iguala las ayudas para servicios sociales que recibe Canarias con la media estatal.

- UPN logró movilizar 50 millones de euros para inversiones en la Comunidad Foral de Navarra, principalmente dirigidas a carreteras, el abaratamiento de peajes e instalaciones deportivas, mientras que los regionalistas asturianos de Foro pactaron un incremento de 53 millones de las inversiones previstas en su comunidad (garantía del ancho europeo y los requisitos de alta velocidad entre Madrid y Asturias, aumento en 20 millones de los costes para reducir emisiones de centrales térmicas y de 2,6 millones de la partida destinada a Asturias para la extensión de la banda ancha, así como 5 millones para los fondos mineros).

- El PNV pactó con el Gobierno, a modo de principal contrapartida por respaldar los PGE para 2017, la modificación del Concierto Económico y la nueva metodología de señalamiento del Cupo para el quinquenio 2017-2021, en virtud del cual el País Vasco abonará al Estado 225 millones de eurosde lo que estaba fijado en función del anterior acuerdo firmado en $2007^{27}$. Asimismo, el acuerdo por el "sí" de los diputados peneuvistas en abril de 2018 a los Presupuestos de ese año incluyó la alta velocidad y la llegada soterrada del tren a las capitales vascas, la reducción de la tarifa eléctrica a la industria vasca o la renovación generacional de la Ertzaintza, entre otras concesiones.

g) En fin, el 1 de junio de 2018 el Congreso de los Diputados aprobó la moción de censura presentada por el Grupo Parlamentario Socialista contra el Gobierno de Rajoy -la primera de la democracia y la segunda presentada en la legislatura ${ }^{28}$, con el concurso decisivo de los partidos nacionalistas, independentistas y regionalistas, ya que entre los 180 votos a favor de la moción se encuentran los de ERC (9), $\operatorname{PDeCAT}^{29}$ (8) PNV (5), Compromís (4), EH Bildu (2) y NC (1), mientras que el diputado de CC se abstuvo. Aunque el nuevo Gobierno de Pedro Sánchez asegura que no ha pactado ninguna concesión a dichos partidos, determinados

27. El Cupo es la aportación que hace el País Vasco cada año a las arcas comunes del Estado por las competencias no transferidas al Gobierno autonómico, como infraestructuras -aeropuertos, puertos, ferrocarriles-, defensa y representación exterior. Con este dinero se contribuye también al mantenimiento de las instituciones comunes, como el Congreso y el Senado.

28. Desde la entrada en vigor de la Constitución en 1978, en España ha habido cuatro mociones de censura, pero sólo la última de ellas -la mencionada en el texto- ha salido adelante:

Ia I980-Moción de censura al Gobierno de Adolfo Suárez. En mayo de 1980, el PSOE, con Felipe González a la cabeza, presentó la primera moción de censura de la historia de la democracia española. Fue contra el Gobierno de Adolfo Suárez, de la ya desaparecida UCD. Suárez tuvo el respaldo de sus diputados, con I66 votos en contra, frente a los I 52 a favor de la moción y 21 abstenciones.

$2^{\underline{a}} \quad$ 1987-Moción de censura al Gobierno de Felipe González. En marzo de 1987, APpresentó una moción de censura contra el Gobierno socialista de Felipe González. El candidato propuesto por AP, Antonio Hernández Mancha, no tuvo el apoyo de la Cámara: sólo 67 votos a favor de la moción frente a los 195 en contra y 70 abstenciones.

$3^{\mathrm{a}} \quad$ 2017-Moción de censura al Gobierno de Mariano Rajoy. El I3 de junio de 2017, UP, liderado por Pablo Iglesias, propuso la tercera moción de censura de la España democrática y la primera contra el Gobierno popular de Mariano Rajoy, que fue rechazada el día siguiente con 170 votos en contra, 82 a favor y 97 abstenciones.

$4^{\underline{a}} \quad$ 2018-Moción de censura al Gobierno de Mariano Rajoy. El 25 de mayo de 2018, el Grupo Parlamentario Socialista presentó la segunda moción de censura contra el Gobierno de Rajoy. Según el escrito presentado a la Mesa del Congreso de los Diputados, esta moción ha sido motivada por la Sentencia de la Sala de lo Penal de la Audiencia Nacional del conocido como "caso Gürtel”. Fue aprobada el I de junio de 2018 con I80 votos a favor, I69 votos en contra y I abstención, dando lugar a la dimisión del Gobierno de Mariano Rajoy del Gobierno y a la investidura como nuevo Presidente del Ejecutivo del candidato propuesto en la moción, el líder socialista Pedro Sánchez.

29. Partit Demòcrata Europeu Català. 
anuncios, medidas y gestos parecen querer desmentirle, máxime cuando el Ejecutivo cuenta con un escaso apoyo parlamentario de 84 diputados para agotar, como es su intención, la legislatura. A modo de muestra:

- El Gobierno ha mantenido los Presupuestos Generales de 2018 pactados en su día por el PP, condición impuesta por el PNV para respaldar la moción y ha manifestado su intención de estudiar el acercamiento de presos de ETA a cárceles del País Vasco. Mientras que el PNV no oculta su propósito de incluir en la reforma del Estatuto vasco que se tramita en la Cámara vasca nuevas competencias (Seguridad Social, prisiones, capacidad para regular consultas ciudadanas o referendos, etc.).

- El Gobierno ha ordenado el traslado de políticos catalanes en prisión provisional a cárceles de Cataluña y ha activado la comisión bilateral Estado-Generalidad para tratar asuntos como la recuperación de la disposición adicional tercera del Estatuto (anulada en su día por el TC, según la cual la inversión del Estado en infraestructuras de Cataluña, excluido el Fondo de Compensación Interrterritorial, será en función del peso que tenga el PIB de esta comunidad en el PIB español) o la retirada de los recursos de inconstitucionalidad interpuestos en su día por el Gobierno contra leyes aprobadas por el Parlamento de Cataluña (sobre pobreza energética, cambio climático, universalidad de la sanidad y protección del derecho a la vivienda) ${ }^{30}$.

\section{LA REFORMA DEL SISTEMA ELECTORAL DE LA REGIÓN DE MURCIA.}

\subsection{Consecuencias del singular sistema electoral murciano vigente hasta julio de 2015 para los partidos minoritarios.}

El sistema electoral diseñado en la Ley 2/1987, de 24 de febrero, Electoral de la Región de Murcia constituía en sí mismo una singularidad dentro de los sistemas autonómicos, pues en una comunidad uniprovincial aquella ley fijaba en cinco el número de circunscripciones electorales infraprovinciales (Valle del Guadalentín, Campo de Cartagena y Mar Menor, Vega del Segura, Noroeste y Río Mula y Altiplano) ${ }^{31}$, además de establecer una barrera electoral del $5 \%$ del total de votos emitidos que, además, no se proyectaba por circunscripción sino para todo el ámbito regional ${ }^{32}$, dando lugar a una clara desproporcionalidad en el reparto de escaños entre las distintas fuerzas políticas y al desigual valor del voto de los ciudadanos ${ }^{33}$.

A lo largo de las nueve elecciones a la Asamblea Regional celebradas hasta la fecha,

30. También se ha especulado sobre si en el seno de la comisión bilateral, acordada finalmente para el I de agosto, se hablaría o no de la convocatoria de un referéndum de autodeterminación y la situación de los presos, dada la redacción final del sexto punto del orden del día: “Consideraciones de la Generalitat sobre la situación política en Catalunya: En materia de derechos y libertades. En relación con las vías de participación ciudadana en las decisiones sobre su futuro político". Vid., "El Gobierno escuchará a la Generalitat sobre presos y referéndum sin comprometerse" (26/o7/2018). Recuperado de https://www.elperiodico.com.

31. El Principado de Asturias es la otra comunidad uniprovincial que, junto a Murcia, optó por circunscripciones infraprovinciales. Tras las elecciones autonómicas de 20I5, PSOE, IU y C's presentaron conjuntamente una proposición de ley de reforma electoral. Los principios que inspiran ésta y otras propuestas de reforma de los sistemas electorales autonómicos pueden verse en García Mahamut, R. (2018): "La reforma de los sistemas electorales autonómicos tras las elecciones de 24 de mayo de 2015 : vectores políticos y jurídicos”, en Teoría y Realidad Constitucional, núm. 4I, pp. I85-2I2. 32. Asturias distingue tres distritos infraprovinciales, pero exige un $3 \%$ de votos por circunscripción, no regional.

33. Vid., por todos, Sierra Rodríguez, J. (2017): El sistema electoral de la Región de Murcia: regulación, balance (1983-2015) y perspectivas. Madrid: Dykinson. 
han concurrido una media de 10 formaciones políticas, aunque con importantes variaciones entre elecciones (6 fuerzas políticas concurrieron en 1983, 8 en 1987, 1991 y 2007, 9 en 1995 y 2003, 10 en 1999 y 16 en 2011 y 2015), si bien algunas de ellas -especialmente los partidos políticos pequeños y otros ligados a espacios territoriales concretos- sólo concurrieron en determinadas circunscripciones.

En todo caso, los tres principales partidos, PP, PSOE e IU, se han presentado en todas las circunscripciones en todos los comicios y han obtenido representación en la Asamblea Regional, con la sola excepción de las elecciones de 2015 en las que IU, que se presentó bajo la coalición "Ganar La Región de Murcia", no obtuvo escaño ${ }^{34}$. Y los nuevos partidos nacionales emergentes, Podemos y C's, irrumpieron en la Asamblea Regional tras las elecciones de 2015 .

Si atendemos a los resultados, podemos distinguir tres períodos bajo dicho sistema: (i) Predominio socialista (1983-1991). El PSOE logra la mayoría absoluta en las elecciones de 1983, 1987 y 1991, con 26, 25 y 24 escaños, respectivamente ${ }^{35}$; (ii) Predominio popular 1995-2011. El PP consigue la mayoría absoluta en las elecciones de 1995, 1999, 2003, 2007 y 2011, con 26, 26, 28, 29 y 33 escaños, respectivamente. (iii) Un nuevo escenario político fragmentado.

En las elecciones de mayo de 2015 ningún partido obtiene mayoría absoluta (PP logra 22 escaños y PSOE 13), y Podemos y C's obtienen 6 y 4 escaños, respectivamente.

La singularidad del sistema electoral murciano ha favorecido sin duda el bipartidismo, por cuanto tiende a sobrerrepresentar a los principales partidos en detrimento de los más pequeños (Barreda y Borge, 2006) ${ }^{36}$ o minoritarios, que han quedado excluidos en diferentes elecciones por no haber superado la exigente barrera electoral regional del $5 \%$, y elloa pesar de haber podido obtener escaño en su respectiva circunscripción. Tres casos son ilustrativos de ello:

a) El Partido Cantonal (PCAN) ${ }^{37}$ no obtuvo escaño en 1983 y 1987, a pesar de tener votos suficientes para ello en la circunscripción dos (Campo de Cartagena y Mar Menor), porque su porcentaje de voto regional $(2,8 \%$ y $3,4 \%$, respectivamente), no superó la mencionada barrera.

b) UPyD, pese a obtener en las elecciones de 2011 el 5,4\% de los votos en la circunscripción tres (Vega del Segura), no accedió al reparto de escaños porque a nivel regional obtuvo un $4,6 \%$ de los sufragios emitidos.

\footnotetext{
34. El Centro Democrático y Social (CDS) se presentó a las elecciones autonómicas en el periodo 1983-2003, estando en coalición en las elecciones de 1995 y 1999 con Unión Centrista (UC). Con un número de votos residual en 1983 , llegó a obtener tres diputados regionales en 1987, pero en las siguientes elecciones de I991 no tuvo representación y posteriormente fue apoyado por un número de electores decreciente. El CDS como organización decidió en 200 s integrarse en el PP, motivo por el que no se presentaría a las siguientes autonómicas en la Región de Murcia. Los nuevos partidos, Podemos y C's, concurrieron por primera vez a los comicios de 2015 y lograron 6 y 4 escaños, respectivamente.

35. El 8 de mayo de 1983 se celebraron las primeras elecciones a la Asamblea Regional bajo las previsiones de la Disposición Transitoria Primera del Estatuto de Autonomía murciano, y en ellas se eligieron a 43 diputados, que pasarían a ser 45 una vez promulgada la ley electoral regional de 1987.

36. Barreda, M. y Botella, J. (2006): La democracia española: realidades y desafíos. Análisis del sistema político español. Barcelona: UOC, p. 273.

37. Partido local cuyo fin primordial es la consecución de la provincia de Cartagena.
} 
c) Y en las elecciones de 2015, la coalición “Ganar La Región de Murcia”38 quedó fuera del reparto de escaños, también en la circunscripcióntres, por haber obtenido el 4,8\% regional, a pesar de que superó con votos al último partido que obtuvo escaño por esa circunscripción ${ }^{39}$.

\subsection{La reforma de 2015.}

El sistema implantado por la Ley 2/1987 fue cuestionado desde el primer momento tanto por la doctrina como por los partidos políticos representados en la Asamblea Regional. El nuevo escenario político tras las elecciones autonómicas de mayo de 2015, en el que ningún partido alcanzó la mayoría absoluta, permitió llevar a efecto la visión compartida en la oposición sobre los elementos mínimos y sustantivos que debían ser objeto de reforma electoral, que fue operada finalmente por la Ley 14/2015, de 28 de julio, aprobada por unanimidad de la Asamblea.

Esta ley, además de rebajar la barrera electoral del $5 \%$ al $3 \%$ y reducirlas cinco circunscripciones a una única circunscripción, incluye otras novedades que afectan al proceso electoral, como la obligación de realizar debates electorales durante el período de campaña electoral, la ampliación de las incompatibilidades -entre ellas, la de los miembros de corporaciones locales de los municipios de la Región de Murcia- y otras que afectan a la Administración Electoral -por ejemplo, la habilitación de la Junta Electoral de Murcia para imponer multas de hasta 1200 euros.

Las consecuencias de lamayor proporcionalidad del nuevo sistema electoral se hacen patentes cuando comprobamos los cambios sustanciales en la composición de la Asamblea Regional de Murcia resultantes de aplicar aquél a los resultados electorales de los comicios autonómicos de 2011 y 2015 (Tablas 4 y 5).

Tabla 4. Datos reales y simulación de escaños con resultados elecciones 2011

\begin{tabular}{|l|l|l|l|l|}
\hline Partido & PP & PSOE & IU & UPyD \\
\hline Datos reales & 33 & 11 & 1 & 0 \\
\hline Simulación & 29 & 11 & 3 & 2 \\
\hline
\end{tabular}

Fuente: Sierra (2017)

Tabla 5. Datos reales y simulación de escaños con resultados elecciones 2015

\begin{tabular}{|l|l|l|l|l|l|}
\hline Partido & PP & PSOE & Podemos & C's & Ganar RM \\
\hline Datos reales & 22 & 13 & 6 & 4 & \\
\hline Simulación & 19 & 12 & 6 & 6 & 2 \\
\hline
\end{tabular}

Fuente: Sierra (2017)

38. Que integraba a Izquierda Unida.

39. Cfr. Sierra Rodríguez, J.: El sistema electoral...op.cit., pp. I2I-I29. 


\section{CONCLUSIONES.}

No cabe duda de que la creciente toma de conciencia ciudadana sobre la posición de ventaja deque gozan los partidos nacionalistas y regionalistas en un Congreso de los Diputados fragmentado con Gobiernos en minoría, que les ha llevado, como nos hemos esmerado en demostrar en esta investigación, a ser decisivos -y, en ocasiones, a ser decisores- y a obtener un enorme rédito político y económico por ese pequeño pero determinante número de votos que aportan, unido a la mayor proporcionalidad del nuevo sistema electoral de la Región de Murcia tras su reforma en julio de 2015, pueden ser factores clave para que el regionalismo logre tener un espacio en el sistema de partidos de una comunidad, la Región de Murcia, tradicionalmente reacia a ello.

Es cierto que Murcia no ha sido nunca una autonomía con demasiado sentimiento "identitario" 40 . En efecto, cada vez que el Centro de Investigaciones Sociológicas (CIS) ha realizado estudios sobre el sentimiento de pertenencia a una región concreta, la Región de Murcia ha estado entre las Comunidades Autónomas con más porcentaje de ciudadanos que reconocen estar orgullosos de ser españoles, tanto o más como murcianos. Sin ir más lejos, en el barómetro postelectoral que el CIS realizó en 2015, tras las elecciones autonómicas de mayo de dicho año, más de un $13 \%$ de los encuestados de la Región aseguró sentirse únicamente español o más español que murciano, aunque la inmensa mayoría, un $83 \%$, declaró sentirse tan murciano como español, lo que dejó en un escaso $5 \%$ el porcentaje de ciudadanos de esta tierra que no se sienten españoles. Quizá esa sea la causa de que no hayan tenido éxito electoral las iniciativas de carácter regionalista, que tampoco han proliferado en demasía en los últimos cuarenta años ${ }^{41}$.

Sin embargo, los años de profunda crisis económica, la corrupción que ha afectado a los partidos tradicionales y, sobre todo, la sensación de agravio que pueden sentir los ciudadanos de la Región al ver cómo otras Comunidades Autónomas gozan de mayor financiación y mejores servicios públicos e infraestructuras,junto a la valoración negativa que la ciudadanía hace en general de la gestión que los responsables políticos regionales de distinto signo han realizado en asuntos específicos (como el déficit hidrológico, la recuperación del Mar Menor o el soterramiento de las vías del tren a su paso por algunas localidades), han sembrado en los últimos tiempos el caldo de cultivo de un incipiente sentimiento regionalista -en ningún caso nacionalista- que amenaza con tener continuidad electoral y quitar miles de votos a las grandes formaciones de corte estatal.

Conviene aclarar que aquella sensación de agravio no es exclusiva de los murcianos, pues mientras que cerca de las tres cuartas partes de los españoles suelen identificarse en los sucesivos estudios con la idea de España como "mi nación, mi país", la evolución de la opinión pública demuestra que las Comunidades Autónomas son convenientes, pero que su funcionamiento deja mucho que desear, sobre todo porque el sistema ha beneficiado

40. Un interesante estudio sobre la conciencia regional puede verse en Ferre Abellán, L. (2015): Las élites políticas y la construcción de la conciencia regional murciana, 1976-1983 (Tesis Doctoral). UNED.

4I. Partido Regionalista Murciano, Partido Murcianista, Nueva Murcia y Unión de los Pueblos de Murcia, entre otros. Sin embargo, el partido Movimiento Ciudadano de Cartagena (MC), fundado en diciembre de 2002, sí ha obtenido representación en el Ayuntamiento de ese municipio: en las primeras elecciones a las que se presentó, en 2003, obtuvo un concejal; en la siguiente legislatura (2007-20II) contó con dos concejales; en la siguiente cita electoral (20II) sólo obtuvo un concejal; y en los comicios de 2015 logró cinco concejales, convirtiéndose su líder, José López, en virtud de un pacto con PSOE y Podemos, en Alcalde de Cartagena hasta junio de 2017. 
a unas provincias o comunidades a costa de otras. Las mencionadas más frecuentemente son Cataluña y el País Vaco. Lo cual coincide con la opinión generalizada -que, además, responde a una realidad objetiva- de que su nivel de vida es más alto que en las demás, y la desigualdad, más acentuada ${ }^{42}$.

Resulta, además, revelador que, según una encuesta de octubre de 2017 encargada por un diario regional en octubre de 2017 -en un momento de profunda crisis institucional provocada por la dimisión del presidente de la Comunidad, el popular Pedro Antonio Sánchez, por su imputación y próximo procesamiento en dos casos de presunta corrupción-, los murcianos tengan, por un lado, una imagen muy negativa de la situación política regional y, por otro, no reprueben la inexistencia de mayoría absoluta en la Asamblea Regional sino que, por el contrario, la valoren como una oportunidad para el diálogo y el encuentro ${ }^{43}$. O que un reciente estudio señale que el presidente cántabro, Miguel Ángel Revilla, que preside su comunidad con un partido regionalista, sea el más votado por los murcianos, por delante de líderes de partidos nacionales ${ }^{44}$.

No es de extrañar, pues, que en los últimos años hayan surgido distintas formaciones políticas de orientación localista y regionalista ${ }^{45}$, de entre las que cabe destacar MC Cartagena ${ }^{46}$ y Somos Región ${ }^{47}$, los cuales, a pesar de no obtener ningún escaño en la Asamblea Regional de Murcia en las pasadas elecciones autonómicas celebradas el 26 de mayo de 2019, alcanzaron un nada despreciable porcentaje de voto (el 2,26 \% y 2,04 \% de los votos, respectivamente $^{48}$ ). Asimismo, en los comicios municipales MC Cartagena fue el partido más votado en esa localidad (con el 27,44 \%, obtuvo 8 concejales) ${ }^{49}$ mientras que Somos Región logró 4 concejales en 3 localidades ( 1 en Torre Pacheco, 2 en Blanca y 1 en Ricote $)^{50}$.

Tendremos que esperar a las próximas elecciones generales y, sobre todo, a las

42. Vid., a este respecto, Cazorla, J. (1999): “Unos más iguales que otros”, en Estudios Regionales, núm. 54, pp. 309-314; Moral, F. (1998): Identidad regional y nacionalismo en el Estado de las Autonomías. Madrid: CIS. Y más recientemente, Pérez. F., Cucarella V. /Hernández, L. (2015): Servicios públicos, diferencias territoriales e igualdad de oportunidades. Fundación BBVA-Ivie: Madrid; y Gallego, R. (dir.) 20I6: Descentralización y desigualdad en el Estado autonómico. Tirant lo Blanch: Valencia.

43. Un 49,8\% de los entrevistados califican de mala o muy mala la situación política regional, frente al II, $3 \%$ que piensa que es buena o muy buena, mientras que un $38,5 \%$ declaran que la situación no es ni buena ni mala. Por otra parte, la mayoría de los entrevistados $(54,5 \%)$ consideran muy bien $(13,2 \%)$ o bien $(4 \mathrm{r}, 3 \%)$ que no haya mayoría absoluta en la Cámara autonómica, mientras que sólo a un 22,I \% les parece mal y muy mal y un 23,2\% se muestra indiferente sobre esta situación. Vid., "Los murcianos ven muy negra la política regional y censuran la ausencia de pactos" (29/10/2017). Recuperado de https://www.laverdad.es.

44. Vid., II Encuesta Adecco ¿Qué personaje político te gustaría tener como jefe? Disponible en: https://adecco.es.

45. En 2016 aparecen Juntos por la Región y Alternativa Regional Murciana.

46. Aunque la formación local cantonalista Movimiento Ciudadano de Cartagena fue fundada unos años antes, en 2002. 47. Fundado en 2018 por el ex presidente de la Comunidad Autónoma Alberto Garre, el Barómetro del Centro de Estudios Murcianos de Opinión Pública (CEMOP) de primavera 2018 llegó a otorgarle el $5,6 \%$ de las papeletas y dos diputados, si bien el Barómetro CEMOP de otoño redujo la estimación directa de voto de ese partido al 2,4\%, sin otorgarles representación parlamentaria. Los Barómetros CEMOP se encuentran disponibles en: http://www.cemopmurcia.es.

48. MC Cartagena concurrió a estas elecciones integrada en una coalición de partidos municipalistas, MC-CDD.

49. Cuando se daba casi por seguro que gobernaría en minoría Movimiento Ciudadano, un polémico acuerdo de última hora entre PP, PSOE y Ciudadanos impidió el acceso de aquél al gobierno municipal.

5o. Por lo que se refiere a las elecciones generales del 28A, MC Cartagena no concurrió, y los regionalistas de Somos Región no obtuvieron representación en Cortes (su porcentaje de voto en el Congreso fue del $0,65 \%$, alcanzando sus tres candidatos al Senado el I, $28 \%, 0,85 \%$ y $0,72 \%)$. 
autonómicas de 2023 para saber si el regionalismo fructificará, por fin, en la Comunidad Autónoma de Murcia.

\section{REFERENCIAS BIBLIOGRÁFICAS}

BARREDA, M. Y BOTELLA, J. (2006): La democracia española: realidades y desafios. Análisis del sistema político español, UOC, p. 273.

CAZORLA, J. (1999): “Unos más iguales que otros”, en Estudios Regionales, núm. 54, pp. 309-314.

FERRE ABELLÁN, L. (2015): Las élites politicas y la construcción de la conciencia regional murciana, 1976-1983 (Tesis Doctoral), UNED.

GALlEGO, R. (dir.) (2016): Descentralización y desigualdad en el Estado autonómico, Tirant lo Blanch.

GÁLVEZ MUÑOZ, L. (2004): "El régimen electoral de Murcia”, en Cuadernos de Derecho Público, núms. 22-23, 2004, pp. 402-404.

GARCÍA ESCUDERO, J. (2016): “El sistema electoral español, su rendimiento y la actual conveniencia de su reforma", comunicación presentada al XII Congreso Español de Sociología "Grandes transformaciones sociales, nuevos desafíos para la Sociología”, Gijón, 30 de junio, 1 y 2 de julio de 2016, pp. 25-29.

GARCÍA MAHAMUT, R., (2018): "La reforma de los sistemas electorales autonómicos tras las elecciones de 24 de mayo de 2015: vectores políticos y jurídicos”, en Teoría y Realidad Constitucional, núm. 41, pp. 185-212. https://doi.org/10.5944/trc.41.2018.22138

MONTERO, J.R. Y RIERA, P. (2009): "El sistema electoral español: cuestiones de desproporcionalidad y de reforma", en Anuario de la Facultad de Derecho de la Universidad Autónoma de Madrid, núm. 13, 2009, p. 234.

MONTERO, J.R.; FERNÁNDEZ ESQUER, C. Y RAMA, J. (2016): “Sistema electoral y votos desperdiciados: ¿votando a ciegas?”. Recuperado de: https://politikon.es.

MORAL, F. (1998): Identidad regional y nacionalismo en el Estado de las Autonomías, 1998, CIS.

OCAÑA, F.A. Y OÑATE RUBALCABAP. (1999): “Índices e indicadores del sistema electoral y del sistema de partidos: una propuesta informática para su cálculo", en Revista Española de Investigaciones Sociológicas, pp. 223-246. https://doi.org/10.2307/40184151 
OLAZABAL, P. (2016): “Se benefician los partidos nacionalistas de la ley electoral?”. Recuperado de: https://limonenelmarmol.wordpress.com.

PANIAGUA, J.L. (2008): “Representación y peso político de los partidos nacionalistas en las Cortes Generales", en Temas para el debate, núm. I62 (Ejemplar dedicado a: Democracia y legislación electoral), pp. 54-56.

PÉREZ. F., CUCARELLA V. Y HERNÁNDEZ, L. (2015): Servicios públicos, diferencias territoriales e igualdad de oportunidades, Fundación BBVA-Ivie: Madrid.

SIERRA RODRÍGUEZ, J. (2017): El sistema electoral de la Región de Murcia: regulación, balance (1983-2015) y perspectivas, Dykinson.

TENA ARREGUI, R. (2015): “El sistema electoral”. Serie sobre la reforma constitucional (IV). Recuperado de https://hayderecho.com 